\title{
Time Scales Delta Iyengar-Type Inequalities
}

\author{
George A. Anastassiou \\ University of Memphis \\ Department of Mathematical Sciences \\ Memphis, TN 38152, U.S.A.
}

\begin{abstract}
Here we give the necessary background on delta time scales approach. Then we present general related time scales delta Iyengar type inequalities for all basic norms. We finish with applications to specific time scales like $\mathbb{R}, \mathbb{Z}$ and $q^{\overline{\mathbb{Z}}}, q>1$.
\end{abstract}

AMS Subject Classifications: 26D15, 39A12, 93C70.

Keywords: Iyengar inequality, time scale, delta calculus.

\section{Introduction}

We are motivated by the following famous Iyengar inequality (1938), [11].

Theorem 1.1. Let $f$ be a differentiable function on $[a, b]$ and $\left|f^{\prime}(x)\right| \leq M$. Then

$$
\left|\int_{a}^{b} f(x) d x-\frac{1}{2}(b-a)(f(a)+f(b))\right| \leq \frac{M(b-a)^{2}}{4}-\frac{(f(b)-f(a))^{2}}{4 M} .
$$

We present generalized analogs of (1.1) to time scales. Motivation comes also from $[2-4,6,7]$.

\section{Background}

Here basics on time scales come from [3, 4, 6-8]. We need the following definition.

Definition 2.1. A time scale is an arbitrary nonempty closed subset of the real numbers, e.g. $\mathbb{R}, \mathbb{Z}, q^{\mathbb{N}_{0}}=\left\{q^{k} \mid k \in \mathbb{N}_{0}=\mathbb{N} \cup\{0\}, q>1\right\}$.

Received January 2, 2019; Accepted July 1, 2019

Communicated by Martin Bohner 
Definition 2.2. If $\mathbb{T}$ is a time scale, then we define the forward jump operator $\sigma: \mathbb{T} \longmapsto$ $\mathbb{T}$ by $\sigma(t)=\inf \{s \in \mathbb{T} \mid s>t\}, \forall t \in \mathbb{T}$; the backward jump operator $\rho: \mathbb{T} \longmapsto \mathbb{T}$ by $\rho(t)=\sup \{s \in \mathbb{T} \mid s<t\}, \forall t \in \mathbb{T}$; and the graininess function $\mu: \mathbb{T} \rightarrow \mathbb{R}_{+}=[0, \infty)$, by $\mu(t)=\sigma(t)-t, \forall t \in \mathbb{T}$. Furthermore for a function $f: \mathbb{T} \rightarrow \mathbb{R}$, we define $f^{\sigma}(t)=f(\sigma(t)), \forall t \in \mathbb{T}$; and $f^{\rho}(t)=f(\rho(t)), \forall t \in \mathbb{T}$.

In this definition we use $\inf \emptyset=\sup \mathbb{T}$ (i.e., $\sigma(t)=t$ if $t$ is the maximum of $\mathbb{T}$ ) and $\sup \emptyset=\inf \mathbb{T}$ (i.e., $\rho(t)=t$ if $t$ is the minimum of $\mathbb{T}$ ).

We call $t \in \mathbb{T}$ right-scattered if $t<\sigma(t), t \in \mathbb{T}$ right-dense if $t=\sigma(t), t \in \mathbb{T}$ leftscattered if $\rho(r)<t, t \in \mathbb{T}$ left-dense if $\rho(t)=t, t \in \mathbb{T}$ isolated if $\rho(t)<t<\sigma(t)$, $t \in \mathbb{T}$ dense if $\rho(t)=t=\sigma(t)$.

We notice that $\rho$ is an increasing function, so is $\rho^{2}(t)=\rho(\rho(t)), \ldots$, so that $\rho^{n}(t)=\rho\left(\rho^{n-1}(t)\right)$ is increasing in $t$ for $n \in \mathbb{N}$. Since $\mathbb{T}$ is closed subset of $\mathbb{R}$ we have that $\sigma(t), \rho(t) \in \mathbb{T}$, for $t \in \mathbb{T}$.

Definition 2.3 (See [8]). A function $f: \mathbb{T} \rightarrow \mathbb{R}$ is called rd-continuous (denoted by $\left.C_{r d}\right)$ if it is continuous at right-dense points of $\mathbb{T}$ and its left-sided limits are finite at left-dense points of $\mathbb{T}$.

If $\mathbb{T}=\mathbb{R}$, then $f: \mathbb{R} \rightarrow \mathbb{R}$ is rd-continuous iff $f$ is continuous. Also, if $\mathbb{T}=\mathbb{Z}$, then any function defined on $\mathbb{Z}$ is rd-continuous ( [9]).

Definition 2.4 (See [8]). If $\sup \mathbb{T}<\infty$ and $\sup \mathbb{T}$ is left-scattered, we let $\mathbb{T}^{k}:=\mathbb{T}-$ $\{\sup \mathbb{T}\}$, otherwise we let $\mathbb{T}^{k}:=\mathbb{T}$ the time scale.

In summary, $\mathbb{T}^{k}=\left\{\begin{array}{l}\mathbb{T}-(\rho(\sup \mathbb{T}), \sup \mathbb{T}], \text { if } \sup \mathbb{T}<\infty \\ \mathbb{T}, \quad \text { if } \sup \mathbb{T}=\infty .\end{array}\right.$

Definition 2.5 (See [8]). Assume $f: \mathbb{T} \rightarrow \mathbb{R}$ is a function and let $t \in \mathbb{T}^{k}$. Then we define $f^{\Delta}(t)$ to be the number (provided it exists) with the property that given any $\varepsilon>0$, there is a neighborhood $U$ of $t$ such that

$$
\left|[f(\sigma(t))-f(s)]-f^{\Delta}(t)[\sigma(t)-s]\right| \leq \varepsilon|\sigma(t)-s|, \quad \forall s \in U .
$$

We call $f^{\Delta}(t)$ the delta (or Hilger [10]) derivative of $f$ at $t$. If $\mathbb{T}=\mathbb{R}$, then $f^{\Delta}=$ $f^{\prime}$, whereas if $\mathbb{T}=\mathbb{Z}$, then $f^{\Delta}(t)=\Delta f(t)=f(t+1)-f(t)$, the usual forward difference operator.

Theorem 2.6 (Existence of Antiderivatives, see [8]). Let $f$ be rd-continuous. Then $f$ has an antiderivative $F$ satisfying $F^{\Delta}=f$.

Definition 2.7 (See [8]). If $f$ is rd-continuous and $t_{0} \in \mathbb{T}$, then we define the integral

$$
F(t)=\int_{t_{0}}^{t} f(\tau) \Delta \tau \text { for } t \in \mathbb{T} .
$$


Therefore for $f \in C_{r d}(\mathbb{T})$ we have by definition

$$
\int_{a}^{b} f(\tau) \Delta \tau=F(b)-F(a)
$$

where $F^{\Delta}=f$.

If $\mathbb{T}=\mathbb{R}$, then

$$
\int_{a}^{b} f(t) \Delta t=\int_{a}^{b} f(t) d t
$$

where the integral on the right hand side is the Riemann integral ( [9]).

If every point in $\mathbb{T}$ is isolated and $a<b$ are in $\mathbb{T}$, then ( [9])

$$
\int_{a}^{b} f(t) \Delta t=\sum_{t=a}^{\rho(b)} f(t) \mu(t) .
$$

Theorem 2.8 (See [8]). Let $f, g$ be $r d$-continuous on $\mathbb{T}, a, b, c \in \mathbb{T}$ and $\alpha, \beta \in \mathbb{R}$. Then
(1) $\int_{a}^{b}(\alpha f(t)+\beta g(t)) \Delta t=\alpha \int_{a}^{b} f(t) \Delta t+\beta \int_{a}^{b} g(t) \Delta t$,
(2) $\int_{a}^{b} f(t) \Delta t=-\int_{b}^{a} f(t) \Delta t$,
(3) $\int_{a}^{b} f(t) \Delta t=\int_{a}^{c} f(t) \Delta t+\int_{c}^{b} f(t) \Delta t$,
(4) $\int_{a}^{b} f(t) g^{\Delta}(t) \Delta t=(f g)(b)-(f g)(a)-\int_{a}^{b} f^{\Delta}(t) g(\sigma(t)) \Delta t$,
(5) $\int_{a}^{a} f(t) \Delta t=0$
(6) $\int_{a}^{b} 1 \Delta t=b-a$.

Theorem 2.9 (Hölder's Inequality, see [2]). Let $a, b \in \mathbb{T}, a \leq b$, and $f, g: \mathbb{T} \rightarrow \mathbb{R}$ be rd-continuous. Then

$$
\int_{a}^{b}|f(t)||g(t)| \Delta t \leq\left(\int_{a}^{b}|f(t)|^{p} \Delta t\right)^{\frac{1}{p}}\left(\int_{a}^{b}|g(t)|^{q} \Delta t\right)^{\frac{1}{q}}
$$

where $p, q>1: \frac{1}{p}+\frac{1}{q}=1$.

Theorem 2.10 (See [8]). Let $f, g \in C_{r d}(\mathbb{T}), a, b \in \mathbb{T}, a \leq b$. Then
1) if $|f(t)| \leq g(t)$ on $[a, b) \cap \mathbb{T}$, then $\left|\int_{a}^{b} f(t) \Delta t\right| \leq \int_{a}^{b} g(t) \Delta t$,
2) if $f(t) \geq 0$, for all $a \leq t<b$ and $t \in \mathbb{T}$, then $\int_{a}^{b} f(t) \Delta t \geq 0$. 
Corollary 2.11 (See [3]). Let $f \in C_{r d}(\mathbb{T}) ; a, b, c \in \mathbb{T}$, with $c \in[a, b] ; f(t) \geq 0, \forall$ $t \in[a, b]$. Then

$$
\int_{a}^{c} f(t) \Delta t \leq \int_{a}^{b} f(t) \Delta t
$$

Definition 2.12 (See [3]). For a function $f: \mathbb{T} \rightarrow \mathbb{R}$ we consider the second derivative $f^{\Delta \Delta}$ provided $f^{\Delta}$ is differentiable on $\mathbb{T}^{k^{2}}=\left(\mathbb{T}^{k}\right)^{k}$ with derivative $f^{\Delta \Delta}=\left(f^{\Delta}\right)^{\Delta}$ : $\mathbb{T}^{k^{2}} \rightarrow \mathbb{R}$. Similarly we define higher order derivatives $f^{\Delta^{n}}: \mathbb{T}^{k^{n}} \rightarrow \mathbb{R}$.

Similarly we define $\sigma^{2}(t)=\sigma(\sigma(t)), \ldots, \sigma^{n}(t)=\sigma\left(\sigma^{n-1}(t)\right), n \in \mathbb{N}$. For convenience we put $\rho^{0}(t)=\sigma^{0}(t)=t, f^{\Delta^{0}}=f, \mathbb{T}^{k^{0}}=\mathbb{T}$.

Notice $\mathbb{T}^{k^{n}} \subset \mathbb{T}^{k^{l}}, l \in\{0,1, \ldots, n\}$.

Denote by $C_{r d}^{n}(\mathbb{T})$ the space of all functions $f \in C_{r d}(\mathbb{T})$ such that $f^{\Delta^{i}} \in C_{r d}(\mathbb{T})$ for $i=1, \ldots, n \in \mathbb{N}$. In this last case $\mathbb{T}^{k}=\mathbb{T}$ is needed.

We need the following Taylor formula.

Theorem 2.13 (Taylor's Formula, see [5,9]). Assume $\mathbb{T}^{k}=\mathbb{T}$ and $f \in C_{r d}^{n}(\mathbb{T}), n \in \mathbb{N}$ and $s, t \in \mathbb{T}$. Here $h_{0}(t, s)=1, \forall s, t \in \mathbb{T} ; k \in \mathbb{N}_{0}$, and

$$
h_{k+1}(t, s)=\int_{s}^{t} h_{k}(\tau, s) \Delta \tau, \quad \forall s, t \in \mathbb{T} .
$$

(then $h_{k}^{\Delta}(t, s)=h_{k-1}(t, s)$, for $k \in \mathbb{N}, \forall t \in \mathbb{T}$, for each $s \in \mathbb{T}$ fixed). Then

$$
f(t)=\sum_{k=0}^{n-1} f^{\Delta^{k}}(s) h_{k}(t, s)+\int_{s}^{t} h_{n-1}(t, \sigma(\tau)) f^{\Delta^{n}}(\tau) \Delta \tau .
$$

Remark 2.14 (to Theorem 2.13). By [9], we have $h_{1}(t, s)=t-s, \forall s, t \in \mathbb{T}$.

So if $t \geq s$ then $h_{1}(t, s) \geq 0, h_{2}(t, s) \geq 0, \ldots, h_{n-1}(t, s) \geq 0$. However for $n$ odd number $h_{n-1}(t, \sigma(\tau)) \geq 0$ for all $s \leq \tau \leq t$ (see [4], p. 635).

Also it holds ( [1])

$$
h_{k}(t, s) \leq \frac{(t-s)^{k}}{k !}, \quad \forall t \geq s, k \in \mathbb{N}_{0} .
$$

Corollary 2.15 (to Theorem 2.13, see [3]). Assume $f \in C_{r d}^{n}(\mathbb{T})$ and $s, t \in \mathbb{T}$. Let $m \in \mathbb{N}$ with $m<n$ Then

$$
f^{\Delta^{m}}(t)=\sum_{k=0}^{n-m-1} f^{\Delta^{k+m}}(s) h_{k}(t, s)+\int_{s}^{t} h_{n-m-1}(t, \sigma(\tau)) f^{\Delta^{n}}(\tau) \Delta \tau .
$$

Proof. Use Theorem 2.13 with $n$ and $f$ substituted by $n-m$ and $f^{\Delta^{m}}$, respectively. 
Corollary 2.16 (See [3]). Let $f \in C_{r d}(\mathbb{T}) ; a, b \in \mathbb{T}$, such that $f(t)>0, \forall t \in[a, b] \cap \mathbb{T}$, then $\int_{a}^{b} f(t) \Delta t>0$.

We mention also the following.

Lemma 2.17 (See [4, p. 631]). Let the time scale $\mathbb{T}$ be such that $\mathbb{T}^{k}=\mathbb{T}$. Let $h_{k}$ : $\mathbb{T}^{2} \rightarrow \mathbb{R}, k \in \mathbb{N}_{0}$, such that $h_{0}(t, s) \equiv 1, \forall s, t \in \mathbb{T}$, and $h_{k+1}(t, s)=\int_{s}^{t} h_{k}(\tau, s) \Delta \tau$, $\forall s, t \in \mathbb{T}$, for all $k \in \mathbb{N}_{0}$.

Then $h_{k}(t, s)$ is continuous in $s \in \mathbb{T}$, for each fixed $t \in \mathbb{T}$; and continuous in $t \in \mathbb{T}$, for each fixed $s \in \mathbb{T}$. Also it holds that $h_{k}(t, \sigma(s))$ is $r d$-continuous in $s \in \mathbb{T}$, for each fixed $t \in \mathbb{T}$; for all $k \in \mathbb{N}_{0}$.

\section{Main Results}

In this article, we assume that $\mathbb{T}^{k}=\mathbb{T}$. Next, we present Iyengar type inequality on time scales for all norms $\|\cdot\|_{p}, 1 \leq p \leq \infty$.

Theorem 3.1. Let $f \in C_{r d}^{n}(\mathbb{T}), n$ is an odd number, $a, b \in \mathbb{T} ; a \leq b$. Here $\sigma$ is continuous and $h_{n-1}(t, s)$ jointly continuous. Then

1)

$$
\begin{gathered}
\left|\int_{a}^{b} f(t) \Delta t-\sum_{k=0}^{n-1}\left(f^{\Delta^{k}}(a) h_{k+1}(x, a)-f^{\Delta^{k}}(b) h_{k+1}(x, b)\right)\right| \leq\left\|f^{\Delta^{n}}\right\|_{\infty,[a, b] \cap \mathbb{T}} \\
{\left[\left(\int_{a}^{x}\left(\int_{a}^{t} h_{n-1}(t, \sigma(\tau)) \Delta \tau\right) \Delta t\right)+\left(\int_{x}^{b}\left(\int_{t}^{b} h_{n-1}(t, \sigma(\tau)) \Delta \tau\right) \Delta t\right)\right],}
\end{gathered}
$$

$\forall x \in[a, b] \cap \mathbb{T}$,

2) assuming $f^{\Delta^{k}}(a)=f^{\Delta^{k}}(b)=0, k=0,1, \ldots, n-1$, we get from (3.1) that

$$
\begin{gathered}
\left|\int_{a}^{b} f(t) \Delta t\right| \leq\left\|f^{\Delta^{n}}\right\|_{\infty,[a, b] \cap \mathbb{T}} \\
{\left[\left(\int_{a}^{x}\left(\int_{a}^{t} h_{n-1}(t, \sigma(\tau)) \Delta \tau\right) \Delta t\right)+\left(\int_{x}^{b}\left(\int_{t}^{b} h_{n-1}(t, \sigma(\tau)) \Delta \tau\right) \Delta t\right)\right],}
\end{gathered}
$$

$\forall x \in[a, b] \cap \mathbb{T}$,

$2{ }_{1}$ ) when $x=a$ we get from (3.2) that

$$
\left|\int_{a}^{b} f(t) \Delta t\right| \leq\left\|f^{\Delta^{n}}\right\|_{\infty,[a, b] \cap \mathbb{T}}\left(\int_{a}^{b}\left(\int_{t}^{b} h_{n-1}(t, \sigma(\tau)) \Delta \tau\right) \Delta t\right),
$$


$2_{2}$ ) when $x=b$ we get from (3.2) that

$$
\left|\int_{a}^{b} f(t) \Delta t\right| \leq\left\|f^{\Delta^{n}}\right\|_{\infty,[a, b] \cap \mathbb{T}}\left(\int_{a}^{b}\left(\int_{a}^{t} h_{n-1}(t, \sigma(\tau)) \Delta \tau\right) \Delta t\right),
$$

$\left.2_{3}\right)$ by (3.3) and (3.4) we get

$$
\begin{gathered}
\left|\int_{a}^{b} f(t) \Delta t\right| \leq\left\|f^{\Delta^{n}}\right\|_{\infty,[a, b] \cap \mathbb{T}} \\
\min \left\{\left(\int_{a}^{b}\left(\int_{t}^{b} h_{n-1}(t, \sigma(\tau)) \Delta \tau\right) \Delta t\right),\left(\int_{a}^{b}\left(\int_{a}^{t} h_{n-1}(t, \sigma(\tau)) \Delta \tau\right) \Delta t\right)\right\},
\end{gathered}
$$

and

3) assuming $f^{\Delta^{k}}(a)=f^{\Delta^{k}}(b)=0, k=1, \ldots, n-1$, by (3.1) we have

$$
\begin{gathered}
\left|\int_{a}^{b} f(t) \Delta t-[f(a)(x-a)+f(b)(b-x)]\right| \leq\left\|f^{\Delta^{n}}\right\|_{\infty,[a, b] \cap \mathbb{T}} \\
{\left[\left(\int_{a}^{x}\left(\int_{a}^{t} h_{n-1}(t, \sigma(\tau)) \Delta \tau\right) \Delta t\right)+\left(\int_{x}^{b}\left(\int_{t}^{b} h_{n-1}(t, \sigma(\tau)) \Delta \tau\right) \Delta t\right)\right],}
\end{gathered}
$$

$\forall x \in[a, b] \cap \mathbb{T}$.

Proof. By [8, p. 23], we have that $\left\|f^{\Delta^{n}}\right\|_{\infty,[a, b] \cap \mathbb{T}}<\infty$. By Theorem 2.13, see (2.2), we have

$$
f(t)-\sum_{k=0}^{n-1} f^{\Delta^{k}}(a) h_{k}(t, a)=\int_{a}^{t} h_{n-1}(t, \sigma(\tau)) f^{\Delta^{n}}(\tau) \Delta \tau
$$

and

$$
f(t)-\sum_{k=0}^{n-1} f^{\Delta^{k}}(b) h_{k}(t, b)=\int_{b}^{t} h_{n-1}(t, \sigma(\tau)) f^{\Delta^{n}}(\tau) \Delta \tau,
$$

$\forall t \in[a, b] \cap \mathbb{T}$.

Then we get

$$
\left|f(t)-\sum_{k=0}^{n-1} f^{\Delta^{k}}(a) h_{k}(t, a)\right| \stackrel{(3.7)}{\leq}\left\|f^{\Delta^{n}}\right\|_{\infty,[a, b] \cap \mathbb{T}} \int_{a}^{t} h_{n-1}(t, \sigma(\tau)) \Delta \tau,
$$

and

$$
\left|f(t)-\sum_{k=0}^{n-1} f^{\Delta^{k}}(b) h_{k}(t, b)\right| \stackrel{(3.8)}{=}\left|\int_{t}^{b} h_{n-1}(t, \sigma(\tau)) f^{\Delta^{n}}(\tau) \Delta \tau\right|
$$




$$
\leq\left(\int_{t}^{b} h_{n-1}(t, \sigma(\tau)) \Delta \tau\right)\left\|f^{\Delta^{n}}\right\|_{\infty,[a, b] \cap \mathbb{T}} .
$$

Therefore it holds by (3.9), (3.10)

$$
\begin{gathered}
-\left\|f^{\Delta^{n}}\right\|_{\infty,[a, b] \cap \mathbb{T}} \int_{a}^{t} h_{n-1}(t, \sigma(\tau)) \Delta \tau \leq f(t)-\sum_{k=0}^{n-1} f^{\Delta^{k}}(a) h_{k}(t, a) \\
\leq\left\|f^{\Delta^{n}}\right\|_{\infty,[a, b] \cap \mathbb{T}} \int_{a}^{t} h_{n-1}(t, \sigma(\tau)) \Delta \tau
\end{gathered}
$$

and

$$
\begin{gathered}
-\left\|f^{\Delta^{n}}\right\|_{\infty,[a, b] \cap \mathbb{T}}\left(\int_{t}^{b} h_{n-1}(t, \sigma(\tau)) \Delta \tau\right) \leq f(t)-\sum_{k=0}^{n-1} f^{\Delta^{k}}(b) h_{k}(t, b) \\
\leq\left\|f^{\Delta^{n}}\right\|_{\infty,[a, b] \cap \mathbb{T}}\left(\int_{t}^{b} h_{n-1}(t, \sigma(\tau)) \Delta \tau\right)
\end{gathered}
$$

$\forall t \in[a, b] \cap \mathbb{T}$.

Consequently, we have

$$
\begin{array}{r}
\sum_{k=0}^{n-1} f^{\Delta^{k}}(a) h_{k}(t, a)-\left\|f^{\Delta^{n}}\right\|_{\infty,[a, b] \cap \mathbb{T}} \int_{a}^{t} h_{n-1}(t, \sigma(\tau)) \Delta \tau \leq f(t) \\
\quad \leq \sum_{k=0}^{n-1} f^{\Delta^{k}}(a) h_{k}(t, a)+\left\|f^{\Delta^{n}}\right\|_{\infty,[a, b] \cap \mathbb{T}} \int_{a}^{t} h_{n-1}(t, \sigma(\tau)) \Delta \tau
\end{array}
$$

and

$$
\begin{aligned}
& \sum_{k=0}^{n-1} f^{\Delta^{k}}(b) h_{k}(t, b)-\left\|f^{\Delta^{n}}\right\|_{\infty,[a, b] \cap \mathbb{T}}\left(\int_{t}^{b} h_{n-1}(t, \sigma(\tau)) \Delta \tau\right) \leq f(t) \\
& \quad \leq \sum_{k=0}^{n-1} f^{\Delta^{k}}(b) h_{k}(t, b)+\left\|f^{\Delta^{n}}\right\|_{\infty,[a, b] \cap \mathbb{T}}\left(\int_{t}^{b} h_{n-1}(t, \sigma(\tau)) \Delta \tau\right),
\end{aligned}
$$

$\forall t \in[a, b] \cap \mathbb{T}$.

Let any $x \in[a, b] \cap \mathbb{T}$, then integrating (3.11), (3.12) we obtain:

$$
\begin{gathered}
\sum_{k=0}^{n-1} f^{\Delta^{k}}(a) h_{k+1}(x, a)-\left\|f^{\Delta^{n}}\right\|_{\infty,[a, b] \cap \mathbb{T}}\left(\int_{a}^{x}\left(\int_{a}^{t} h_{n-1}(t, \sigma(\tau)) \Delta \tau\right) \Delta t\right) \\
\leq \int_{a}^{x} f(t) \Delta t \leq
\end{gathered}
$$




$$
\sum_{k=0}^{n-1} f^{\Delta^{k}}(a) h_{k+1}(x, a)+\left\|f^{\Delta^{n}}\right\|_{\infty,[a, b] \cap \mathbb{T}}\left(\int_{a}^{x}\left(\int_{a}^{t} h_{n-1}(t, \sigma(\tau)) \Delta \tau\right) \Delta t\right),
$$

and

$$
\begin{gathered}
-\sum_{k=0}^{n-1} f^{\Delta^{k}}(b) h_{k+1}(x, b)-\left\|f^{\Delta^{n}}\right\|_{\infty,[a, b] \cap \mathbb{T}}\left(\int_{x}^{b}\left(\int_{t}^{b} h_{n-1}(t, \sigma(\tau)) \Delta \tau\right) \Delta t\right) \\
\leq \int_{x}^{b} f(t) \Delta t \leq \\
-\sum_{k=0}^{n-1} f^{\Delta^{k}}(b) h_{k+1}(x, b)+\left\|f^{\Delta^{n}}\right\|_{\infty,[a, b] \cap \mathbb{T}}\left(\int_{x}^{b}\left(\int_{t}^{b} h_{n-1}(t, \sigma(\tau)) \Delta \tau\right) \Delta t\right) .
\end{gathered}
$$

Adding (3.13) and (3.14) we derive

$$
\begin{gathered}
\sum_{k=0}^{n-1}\left(f^{\Delta^{k}}(a) h_{k+1}(x, a)-f^{\Delta^{k}}(b) h_{k+1}(x, b)\right)-\left\|f^{\Delta^{n}}\right\|_{\infty,[a, b] \cap \mathbb{T}} \cdot \\
{\left[\left(\int_{a}^{x}\left(\int_{a}^{t} h_{n-1}(t, \sigma(\tau)) \Delta \tau\right) \Delta t\right)+\left(\int_{x}^{b}\left(\int_{t}^{b} h_{n-1}(t, \sigma(\tau)) \Delta \tau\right) \Delta t\right)\right]} \\
\leq \int_{a}^{b} f(t) \Delta t \leq \\
{\left[\sum_{k=0}^{n-1}\left(f^{\Delta^{k}}(a) h_{k+1}(x, a)-f^{\Delta^{k}}(b) h_{k+1}(x, b)\right)+\left\|f^{\Delta^{n}}\right\|_{\infty,[a, b] \cap \mathbb{T}} \cdot\right.} \\
{\left[\left(\int_{a}^{x}\left(\int_{a}^{t} h_{n-1}(t, \sigma(\tau)) \Delta \tau\right) \Delta t\right)+\left(\int_{x}^{b}\left(\int_{t}^{b} h_{n-1}(t, \sigma(\tau)) \Delta \tau\right) \Delta t\right)\right]}
\end{gathered}
$$

$\forall x \in[a, b] \cap \mathbb{T}$.

The proof is now complete.

We continue with the next result.

Theorem 3.2. Let $f \in C_{r d}^{n}(\mathbb{T}), n \in \mathbb{N}$ is odd, $a, b \in \mathbb{T}$; $a \leq b$. Then 1)

$$
\begin{gathered}
\left|\int_{a}^{b} f(t) \Delta t-\sum_{k=0}^{n-1}\left(f^{\Delta^{k}}(a) h_{k+1}(x, a)-f^{\Delta^{k}}(b) h_{k+1}(x, b)\right)\right| \leq \\
\left\|f^{\Delta^{n}}\right\|_{L_{1}([a, b] \cap \mathbb{T})}\left\{\int_{a}^{x}(t-\sigma(a))^{n-1} \Delta t+\int_{x}^{b}(\sigma(b)-t)^{n-1} \Delta t\right\},
\end{gathered}
$$

$\forall x \in[a, b] \cap \mathbb{T}$, 
2) assuming $f^{\Delta^{k}}(a)=f^{\Delta^{k}}(b)=0, k=0,1, \ldots, n-1$, from (3.16) we obtain

$$
\begin{gathered}
\left|\int_{a}^{b} f(t) \Delta t\right| \leq\left\|f^{\Delta^{n}}\right\|_{L_{1}([a, b] \cap \mathbb{T})} . \\
\left\{\int_{a}^{x}(t-\sigma(a))^{n-1} \Delta t+\int_{x}^{b}(\sigma(b)-t)^{n-1} \Delta t\right\},
\end{gathered}
$$

$\forall x \in[a, b] \cap \mathbb{T}$,

21) when $x=a$ by (3.17) we get

$$
\left|\int_{a}^{b} f(t) \Delta t\right| \leq\left\|f^{\Delta^{n}}\right\|_{L_{1}([a, b] \cap \mathbb{T})}\left(\int_{a}^{b}(\sigma(b)-t)^{n-1} \Delta t\right),
$$

$2_{2}$ ) when $x=b$ by (3.17) we get

$$
\left|\int_{a}^{b} f(t) \Delta t\right| \leq\left\|f^{\Delta^{n}}\right\|_{L_{1}([a, b] \cap \mathbb{T})}\left(\int_{a}^{x}(t-\sigma(a))^{n-1} \Delta t\right),
$$

$22_{3}$ ) by (3.18), (3.19) we have

$$
\begin{gathered}
\left|\int_{a}^{b} f(t) \Delta t\right| \leq\left\|f^{\Delta^{n}}\right\|_{L_{1}([a, b] \cap \mathbb{T})} \cdot \\
\min \left\{\left(\int_{a}^{b}(\sigma(b)-t)^{n-1} \Delta t\right),\left(\int_{a}^{b}(t-\sigma(a))^{n-1} \Delta t\right)\right\},
\end{gathered}
$$

3) assuming $f^{\Delta^{k}}(a)=f^{\Delta^{k}}(b)=0, k=1, \ldots, n-1$, by (3.16) we derive

$$
\begin{gathered}
\left|\int_{a}^{b} f(t) \Delta t-[f(a)(x-a)+f(b)(b-x)]\right| \leq \\
\left\|f^{\Delta^{n}}\right\|_{L_{1}([a, b] \cap \mathbb{T})}\left\{\int_{a}^{x}(t-\sigma(a))^{n-1} \Delta t+\int_{x}^{b}(\sigma(b)-t)^{n-1} \Delta t\right\},
\end{gathered}
$$

$\forall x \in[a, b] \cap \mathbb{T}$.

Proof. Clearly, here it holds $\left\|f^{\Delta^{n}}\right\|_{L_{1}([a, b] \cap \mathbb{T})}<\infty$.

Set $h_{0}(t, s)=1, \forall s, t \in \mathbb{T} ; k \in \mathbb{N}_{0}$, and $h_{k+1}(t, s)=\int_{s}^{t} h_{k}(\tau, s) \Delta \tau, \forall s, t \in \mathbb{T}$.

Easily, it holds $\left|h_{n}(t, s)\right| \leq|t-s|^{n}, \forall n \in \mathbb{N}, \forall s, t \in \mathbb{T}$.

By Theorem 2.13 (3) we have

$$
f(t)-\sum_{k=0}^{n-1} f^{\Delta^{k}}(a) h_{k}(t, a)=\int_{a}^{t} h_{n-1}(t, \sigma(\tau)) f^{\Delta^{n}}(\tau) \Delta \tau
$$


and

$$
f(t)-\sum_{k=0}^{n-1} f^{\Delta^{k}}(b) h_{k}(t, b)=\int_{b}^{t} h_{n-1}(t, \sigma(\tau)) f^{\Delta^{n}}(\tau) \Delta \tau,
$$

$\forall t \in[a, b] \cap \mathbb{T}$.

Then

$$
\begin{gathered}
\left|f(t)-\sum_{k=0}^{n-1} f^{\Delta^{k}}(a) h_{k}(t, a)\right|=\left|\int_{a}^{t} h_{n-1}(t, \sigma(\tau)) f^{\Delta^{n}}(\tau) \Delta \tau\right| \leq \\
\int_{a}^{t}\left|h_{n-1}(t, \sigma(\tau))\right|\left|f^{\Delta^{n}}(\tau)\right| \Delta \tau \leq \int_{a}^{t}|t-\sigma(\tau)|^{n-1}\left|f^{\Delta^{n}}(\tau)\right| \Delta \tau \leq \\
(t-\sigma(a))^{n-1}\left\|f^{\Delta^{n}}\right\|_{L_{1}([a, b] \cap \mathbb{T})} .
\end{gathered}
$$

Furthermore we have

$$
\begin{gathered}
\left|f(t)-\sum_{k=0}^{n-1} f^{\Delta^{k}}(b) h_{k}(t, b)\right|=\left|\int_{t}^{b} h_{n-1}(t, \sigma(\tau)) f^{\Delta^{n}}(\tau) \Delta \tau\right| \leq \\
\int_{t}^{b}\left|h_{n-1}(t, \sigma(\tau))\right|\left|f^{\Delta^{n}}(\tau)\right| \Delta \tau \leq \int_{t}^{b}|t-\sigma(\tau)|^{n-1}\left|f^{\Delta^{n}}(\tau)\right| \Delta \tau \leq \\
(\sigma(b)-t)^{n-1}\left\|f^{\Delta^{n}}\right\|_{L_{1}([a, b] \cap \mathbb{T})} .
\end{gathered}
$$

Therefore it holds

$$
\begin{gathered}
-(t-\sigma(a))^{n-1}\left\|f^{\Delta^{n}}\right\|_{L_{1}([a, b] \cap \mathbb{T})} \leq f(t)-\sum_{k=0}^{n-1} f^{\Delta^{k}}(a) h_{k}(t, a) \\
\leq(t-\sigma(a))^{n-1}\left\|f^{\Delta^{n}}\right\|_{L_{1}([a, b] \cap \mathbb{T})}
\end{gathered}
$$

and

$$
\begin{gathered}
-(\sigma(b)-t)^{n-1}\left\|f^{\Delta^{n}}\right\|_{L_{1}([a, b] \cap \mathbb{T})} \leq f(t)-\sum_{k=0}^{n-1} f^{\Delta^{k}}(b) h_{k}(t, b) \\
\leq(\sigma(b)-t)^{n-1}\left\|f^{\Delta^{n}}\right\|_{L_{1}([a, b] \cap \mathbb{T})}
\end{gathered}
$$

$\forall t \in[a, b] \cap \mathbb{T}$.

Consequently it holds

$$
\begin{gathered}
\sum_{k=0}^{n-1} f^{\Delta^{k}}(a) h_{k}(t, a)-(t-\sigma(a))^{n-1}\left\|f^{\Delta^{n}}\right\|_{L_{1}([a, b] \cap \mathbb{T})} \leq f(t) \\
\leq \sum_{k=0}^{n-1} f^{\Delta^{k}}(a) h_{k}(t, a)+(t-\sigma(a))^{n-1}\left\|f^{\Delta^{n}}\right\|_{L_{1}([a, b] \cap \mathbb{T})}
\end{gathered}
$$


and

$$
\begin{aligned}
& \sum_{k=0}^{n-1} f^{\Delta^{k}}(b) h_{k}(t, b)-(\sigma(b)-t)^{n-1}\left\|f^{\Delta^{n}}\right\|_{L_{1}([a, b] \cap \mathbb{T})} \leq f(t) \\
& \quad \leq \sum_{k=0}^{n-1} f^{\Delta^{k}}(b) h_{k}(t, b)+(\sigma(b)-t)^{n-1}\left\|f^{\Delta^{n}}\right\|_{L_{1}([a, b] \cap \mathbb{T})}
\end{aligned}
$$

$\forall t \in[a, b] \cap \mathbb{T}$.

Let any $x \in[a, b] \cap \mathbb{T}$, then integrating by integration we have

$$
\begin{gathered}
\sum_{k=0}^{n-1} f^{\Delta^{k}}(a) h_{k+1}(x, a)-\left(\int_{a}^{x}(t-\sigma(a))^{n-1} \Delta t\right)\left\|f^{\Delta^{n}}\right\|_{L_{1}([a, b] \cap \mathbb{T})} \\
\leq \int_{a}^{x} f(t) \Delta t \leq \\
\sum_{k=0}^{n-1} f^{\Delta^{k}}(a) h_{k+1}(x, a)+\left(\int_{a}^{x}(t-\sigma(a))^{n-1} \Delta t\right)\left\|f^{\Delta^{n}}\right\|_{L_{1}([a, b] \cap \mathbb{T})},
\end{gathered}
$$

and

$$
\begin{gathered}
-\sum_{k=0}^{n-1} f^{\Delta^{k}}(b) h_{k+1}(x, b)-\left(\int_{x}^{b}(\sigma(b)-t)^{n-1} \Delta t\right)\left\|f^{\Delta^{n}}\right\|_{L_{1}([a, b] \cap \mathbb{T})} \\
\leq \int_{x}^{b} f(t) \Delta t \leq \\
-\sum_{k=0}^{n-1} f^{\Delta^{k}}(b) h_{k+1}(x, b)+\left(\int_{x}^{b}(\sigma(b)-t)^{n-1} \Delta t\right)\left\|f^{\Delta^{n}}\right\|_{L_{1}([a, b] \cap \mathbb{T})}
\end{gathered}
$$

$\forall x \in[a, b] \cap \mathbb{T}$.

Adding (3.22) and (3.23), we obtain

$$
\begin{gathered}
\sum_{k=0}^{n-1}\left(f^{\Delta^{k}}(a) h_{k+1}(x, a)-f^{\Delta^{k}}(b) h_{k+1}(x, b)\right)- \\
\left\|f^{\Delta^{n}}\right\|_{L_{1}([a, b] \cap \mathbb{T})}\left\{\left(\int_{a}^{x}(t-\sigma(a))^{n-1} \Delta t\right)+\left(\int_{x}^{b}(\sigma(b)-t)^{n-1} \Delta t\right)\right\} \\
\leq \int_{a}^{b} f(t) \Delta t \leq \\
\sum_{k=0}^{n-1}\left(f^{\Delta^{k}}(a) h_{k+1}(x, a)-f^{\Delta^{k}}(b) h_{k+1}(x, b)\right)+
\end{gathered}
$$




$$
\left\|f^{\Delta^{n}}\right\|_{L_{1}([a, b] \cap \mathbb{T})}\left\{\left(\int_{a}^{x}(t-\sigma(a))^{n-1} \Delta t\right)+\left(\int_{x}^{b}(\sigma(b)-t)^{n-1} \Delta t\right)\right\},
$$

$\forall x \in[a, b] \cap \mathbb{T}$.

The proof is now complete.

We continue with the next result.

Theorem 3.3. Let $f \in C_{r d}^{n}(\mathbb{T})$, $n$ is an odd number, $a, b \in \mathbb{T} ; a \leq b ; p, q>1: \frac{1}{p}+\frac{1}{q}=$ 1 ; $\sigma$ is continuous and $h_{n-1}(t, s)$ is jointly continuous. Then

$$
\begin{aligned}
& \left|\int_{a}^{b} f(t) \Delta t-\sum_{k=0}^{n-1}\left(f^{\Delta^{k}}(a) h_{k+1}(x, a)-f^{\Delta^{k}}(b) h_{k+1}(x, b)\right)\right| \leq\left\|f^{\Delta^{n}}\right\|_{L_{q}([a, b] \cap \mathbb{T})} . \\
& {\left[\left(\int_{a}^{x}\left(\int_{a}^{t} h_{n-1}(t, \sigma(\tau))^{p} \Delta \tau\right)^{\frac{1}{p}} \Delta t\right)+\left(\int_{x}^{b}\left(\int_{t}^{b} h_{n-1}(t, \sigma(\tau))^{p} \Delta \tau\right)^{\frac{1}{p}} \Delta t\right)\right],}
\end{aligned}
$$

$\forall x \in[a, b] \cap \mathbb{T}$,

2) assuming $f^{\Delta^{k}}(a)=f^{\Delta^{k}}(b)=0, k=0,1, \ldots, n-1$, by (3.25) we have that

$$
\begin{gathered}
\left|\int_{a}^{b} f(t) \Delta t\right| \leq\left\|f^{\Delta^{n}}\right\|_{L_{q}([a, b] \cap \mathbb{T})} \cdot \\
{\left[\left(\int_{a}^{x}\left(\int_{a}^{t} h_{n-1}(t, \sigma(\tau))^{p} \Delta \tau\right)^{\frac{1}{p}} \Delta t\right)+\left(\int_{x}^{b}\left(\int_{t}^{b} h_{n-1}(t, \sigma(\tau))^{p} \Delta \tau\right)^{\frac{1}{p}} \Delta t\right)\right]}
\end{gathered}
$$

$\forall x \in[a, b] \cap \mathbb{T}$,

21) when $x=a$ by (3.26) we get

$$
\left|\int_{a}^{b} f(t) \Delta t\right| \leq\left\|f^{\Delta^{n}}\right\|_{L_{q}([a, b] \cap \mathbb{T})}\left(\int_{a}^{b}\left(\int_{t}^{b} h_{n-1}(t, \sigma(\tau))^{p} \Delta \tau\right)^{\frac{1}{p}} \Delta t\right),
$$

$2_{2}$ ) when $x=b$ by (3.26) we get

$$
\left|\int_{a}^{b} f(t) \Delta t\right| \leq\left\|f^{\Delta^{n}}\right\|_{L_{q}([a, b] \cap \mathbb{T})}\left(\int_{a}^{b}\left(\int_{a}^{t} h_{n-1}(t, \sigma(\tau))^{p} \Delta \tau\right)^{\frac{1}{p}} \Delta t\right)
$$

$2_{3}$ ) by (3.27), (3.28) we derive that

$$
\left|\int_{a}^{b} f(t) \Delta t\right| \leq\left\|f^{\Delta^{n}}\right\|_{L_{q}([a, b] \cap \mathbb{T})} .
$$


$\min \left\{\left(\int_{a}^{b}\left(\int_{t}^{b} h_{n-1}(t, \sigma(\tau))^{p} \Delta \tau\right)^{\frac{1}{p}} \Delta t\right),\left(\int_{a}^{b}\left(\int_{a}^{t} h_{n-1}(t, \sigma(\tau))^{p} \Delta \tau\right)^{\frac{1}{p}} \Delta t\right)\right\}$

3) assuming $f^{\Delta^{k}}(a)=f^{\Delta^{k}}(b)=0, k=1, \ldots, n-1$, by (3.25) we obtain

$$
\begin{gathered}
\left|\int_{a}^{b} f(t) \Delta t-[f(a)(x-a)+f(b)(b-x)]\right| \leq\left\|f^{\Delta^{n}}\right\|_{L_{q}([a, b] \cap \mathbb{T})} \\
{\left[\left(\int_{a}^{x}\left(\int_{a}^{t} h_{n-1}(t, \sigma(\tau))^{p} \Delta \tau\right)^{\frac{1}{p}} \Delta t\right)+\left(\int_{x}^{b}\left(\int_{t}^{b} h_{n-1}(t, \sigma(\tau))^{p} \Delta \tau\right)^{\frac{1}{p}} \Delta t\right)\right],}
\end{gathered}
$$

$\forall x \in[a, b] \cap \mathbb{T}$.

Proof. As before, we have

$$
K(t, a):=f(t)-\sum_{k=0}^{n-1} f^{\Delta^{k}}(a) h_{k}(t, a)=\int_{a}^{t} h_{n-1}(t, \sigma(\tau)) f^{\Delta^{n}}(\tau) \Delta \tau,
$$

and

$$
K(t, b):=f(t)-\sum_{k=0}^{n-1} f^{\Delta^{k}}(b) h_{k}(t, b)=\int_{b}^{t} h_{n-1}(t, \sigma(\tau)) f^{\Delta^{n}}(\tau) \Delta \tau,
$$

$\forall t \in[a, b] \cap \mathbb{T}$.

We have that (by use of (2.1))

$$
\begin{aligned}
|K(t, a)| & \leq\left(\int_{a}^{t} h_{n-1}(t, \sigma(\tau))^{p} \Delta \tau\right)^{\frac{1}{p}}\left(\int_{a}^{t}\left|f^{\Delta^{n}}(\tau)\right|^{q} \Delta \tau\right)^{\frac{1}{q}} \\
\leq & \left(\int_{a}^{t} h_{n-1}(t, \sigma(\tau))^{p} \Delta \tau\right)^{\frac{1}{p}}\left\|f^{\Delta^{n}}\right\|_{L_{q}([a, b] \cap \mathbb{T})}
\end{aligned}
$$

and

$$
\begin{gathered}
|K(t, b)|=\left|\int_{t}^{b} h_{n-1}(t, \sigma(\tau)) f^{\Delta^{n}}(\tau) \Delta \tau\right| \leq \\
\left(\int_{t}^{b} h_{n-1}(t, \sigma(\tau))^{p} \Delta \tau\right)^{\frac{1}{p}}\left(\int_{t}^{b}\left|f^{\Delta^{n}}(\tau)\right|^{q} \Delta \tau\right)^{\frac{1}{q}} \\
\leq\left(\int_{t}^{b} h_{n-1}(t, \sigma(\tau))^{p} \Delta \tau\right)^{\frac{1}{p}}\left\|f^{\Delta^{n}}\right\|_{L_{q}([a, b] \cap \mathbb{T})},
\end{gathered}
$$

$\forall t \in[a, b] \cap \mathbb{T}$ 
Hence it holds

$$
\begin{gathered}
-\left(\int_{a}^{t} h_{n-1}(t, \sigma(\tau))^{p} \Delta \tau\right)^{\frac{1}{p}}\left\|f^{\Delta^{n}}\right\|_{L_{q}([a, b] \cap \mathbb{T})} \leq K(t, a) \\
\leq\left(\int_{a}^{t} h_{n-1}(t, \sigma(\tau))^{p} \Delta \tau\right)^{\frac{1}{p}}\left\|f^{\Delta^{n}}\right\|_{L_{q}([a, b] \cap \mathbb{T})}
\end{gathered}
$$

and

$$
\begin{gathered}
-\left(\int_{t}^{b} h_{n-1}(t, \sigma(\tau))^{p} \Delta \tau\right)^{\frac{1}{p}}\left\|f^{\Delta^{n}}\right\|_{L_{q}([a, b] \cap \mathbb{T})} \leq K(t, b) \\
\leq\left(\int_{t}^{b} h_{n-1}(t, \sigma(\tau))^{p} \Delta \tau\right)^{\frac{1}{p}}\left\|f^{\Delta^{n}}\right\|_{L_{q}([a, b] \cap \mathbb{T})},
\end{gathered}
$$

$\forall t \in[a, b] \cap \mathbb{T}$.

That is,

$$
\begin{gathered}
\sum_{k=0}^{n-1} f^{\Delta^{k}}(a) h_{k}(t, a)-\left(\int_{a}^{t} h_{n-1}(t, \sigma(\tau))^{p} \Delta \tau\right)^{\frac{1}{p}}\left\|f^{\Delta^{n}}\right\|_{L_{q}([a, b] \cap \mathbb{T})} \leq f(t) \\
\leq \sum_{k=0}^{n-1} f^{\Delta^{k}}(a) h_{k}(t, a)+\left(\int_{a}^{t} h_{n-1}(t, \sigma(\tau))^{p} \Delta \tau\right)^{\frac{1}{p}}\left\|f^{\Delta^{n}}\right\|_{L_{q}([a, b] \cap \mathbb{T})}
\end{gathered}
$$

and

$$
\begin{gathered}
\sum_{k=0}^{n-1} f^{\Delta^{k}}(b) h_{k}(t, b)-\left(\int_{t}^{b} h_{n-1}(t, \sigma(\tau))^{p} \Delta \tau\right)^{\frac{1}{p}}\left\|f^{\Delta^{n}}\right\|_{L_{q}([a, b] \cap \mathbb{T})} \leq f(t) \\
\leq \sum_{k=0}^{n-1} f^{\Delta^{k}}(b) h_{k}(t, b)+\left(\int_{t}^{b} h_{n-1}(t, \sigma(\tau))^{p} \Delta \tau\right)^{\frac{1}{p}}\left\|f^{\Delta^{n}}\right\|_{L_{q}([a, b] \cap \mathbb{T})}
\end{gathered}
$$

$\forall t \in[a, b] \cap \mathbb{T}$.

Let any $x \in[a, b] \cap \mathbb{T}$, then by integration we get

$$
\begin{gathered}
\sum_{k=0}^{n-1} f^{\Delta^{k}}(a) h_{k+1}(x, a)-\left\|f^{\Delta^{n}}\right\|_{L_{q}([a, b] \cap \mathbb{T})}\left(\int_{a}^{x}\left(\int_{a}^{t} h_{n-1}(t, \sigma(\tau))^{p} \Delta \tau\right)^{\frac{1}{p}} \Delta t\right) \\
\leq \int_{a}^{x} f(t) \Delta t \leq \\
\sum_{k=0}^{n-1} f^{\Delta^{k}}(a) h_{k+1}(x, a)+\left\|f^{\Delta^{n}}\right\|_{L_{q}([a, b] \cap \mathbb{T})}\left(\int_{a}^{x}\left(\int_{a}^{t} h_{n-1}(t, \sigma(\tau))^{p} \Delta \tau\right)^{\frac{1}{p}} \Delta t\right),
\end{gathered}
$$


and

$$
\begin{gathered}
-\sum_{k=0}^{n-1} f^{\Delta^{k}}(b) h_{k+1}(x, b)-\left\|f^{\Delta^{n}}\right\|_{L_{q}([a, b] \cap \mathbb{T})}\left(\int_{x}^{b}\left(\int_{t}^{b} h_{n-1}(t, \sigma(\tau))^{p} \Delta \tau\right)^{\frac{1}{p}} \Delta t\right) \\
\leq \int_{x}^{b} f(t) \Delta t \leq \\
-\sum_{k=0}^{n-1} f^{\Delta^{k}}(b) h_{k+1}(x, b)+\left\|f^{\Delta^{n}}\right\|_{L_{q}([a, b] \cap \mathbb{T})}\left(\int_{x}^{b}\left(\int_{t}^{b} h_{n-1}(t, \sigma(\tau))^{p} \Delta \tau\right)^{\frac{1}{p}} \Delta t\right) .
\end{gathered}
$$

Adding (3.31) and (3.32) we obtain

$$
\begin{gathered}
\sum_{k=0}^{n-1}\left(f^{\Delta^{k}}(a) h_{k+1}(x, a)-f^{\Delta^{k}}(b) h_{k+1}(x, b)\right)- \\
\left\|f^{\Delta^{n}}\right\|_{L_{q}([a, b] \cap \mathbb{T})}\left\{\left(\int_{a}^{x}\left(\int_{a}^{t} h_{n-1}(t, \sigma(\tau))^{p} \Delta \tau\right)^{\frac{1}{p}} \Delta t\right)+\right. \\
\left.\left(\int_{x}^{b}\left(\int_{t}^{b} h_{n-1}(t, \sigma(\tau))^{p} \Delta \tau\right)^{\frac{1}{p}} \Delta t\right)\right\} \\
\sum_{k=0}^{n-1}\left(\int_{a}^{\Delta^{k}}(a) h_{k+1}(x, a)-f^{\Delta^{k}}(b) h_{k+1}(x, b)\right)+ \\
\left\|f^{\Delta^{n}}\right\|_{L_{q}([a, b] \cap \mathbb{T})}\left\{\left(\int_{a}^{x}\left(\int_{a}^{t} h_{n-1}(t, \sigma(\tau))^{p} \Delta \tau\right)^{\frac{1}{p}} \Delta t\right)+\right. \\
\left.\left(\int_{x}^{b}\left(\int_{t}^{b} h_{n-1}(t, \sigma(\tau))^{p} \Delta \tau\right)^{\frac{1}{p}} \Delta t\right)\right\}
\end{gathered}
$$

$\forall x \in[a, b] \cap \mathbb{T}$.

The proof is now complete.

We continue with the next result.

Theorem 3.4. Let $f \in C_{r d}^{n}(\mathbb{T}), m, n \in \mathbb{N}, m<n, n-m$ is odd, $a, b \in \mathbb{T}$; $a \leq b$. Here $\sigma$ is continuous and $h_{n-m-1}(t, s)$ is jointly continuous. Then 
1)

$$
\begin{aligned}
\mid \int_{a}^{b} f^{\Delta^{m}}(t) \Delta t- & \left(\sum_{k=0}^{n-m-1}\left(f^{\Delta^{k+m}}(a) h_{k+1}(x, a)-f^{\Delta^{k+m}}(b) h_{k+1}(x, b)\right)\right) \mid \leq \\
\left\|f^{\Delta^{n}}\right\|_{\infty,[a, b] \cap \mathbb{T}} & {\left[\left(\int_{a}^{x}\left(\int_{a}^{t} h_{n-m-1}(t, \sigma(\tau)) \Delta \tau\right) \Delta t\right)+\right.} \\
& \left.\left(\int_{x}^{b}\left(\int_{t}^{b} h_{n-m-1}(t, \sigma(\tau)) \Delta \tau\right) \Delta t\right)\right]
\end{aligned}
$$

$\forall x \in[a, b] \cap \mathbb{T}$,

2) assuming $f^{\Delta^{k+m}}(a)=f^{\Delta^{k+m}}(b)=0, k=0,1, \ldots, n-m-1$, we get from (3.34) that

$$
\begin{gathered}
\left|\int_{a}^{b} f^{\Delta^{m}}(t) \Delta t\right| \leq\left\|f^{\Delta^{n}}\right\|_{\infty,[a, b] \cap \mathbb{T}} \cdot \\
{\left[\left(\int_{a}^{x}\left(\int_{a}^{t} h_{n-m-1}(t, \sigma(\tau)) \Delta \tau\right) \Delta t\right)+\left(\int_{x}^{b}\left(\int_{t}^{b} h_{n-m-1}(t, \sigma(\tau)) \Delta \tau\right) \Delta t\right)\right],}
\end{gathered}
$$

$\forall x \in[a, b] \cap \mathbb{T}$,

$2{ }_{1}$ ) when $x=a$ we get from (3.35) that

$$
\left|\int_{a}^{b} f^{\Delta^{m}}(t) \Delta t\right| \leq\left\|f^{\Delta^{n}}\right\|_{\infty,[a, b] \cap \mathbb{T}}\left(\int_{a}^{b}\left(\int_{t}^{b} h_{n-m-1}(t, \sigma(\tau)) \Delta \tau\right) \Delta t\right),
$$

$2_{2}$ ) when $x=b$ we get from (3.35) that

$$
\left|\int_{a}^{b} f^{\Delta^{m}}(t) \Delta t\right| \leq\left\|f^{\Delta^{n}}\right\|_{\infty,[a, b] \cap \mathbb{T}}\left(\int_{a}^{b}\left(\int_{a}^{t} h_{n-m-1}(t, \sigma(\tau)) \Delta \tau\right) \Delta t\right)
$$

$2_{3}$ ) by (3.36), (3.37) we get

$$
\left|\int_{a}^{b} f^{\Delta^{m}}(t) \Delta t\right| \leq\left\|f^{\Delta^{n}}\right\|_{\infty,[a, b] \cap \mathbb{T}}
$$

$\min \left\{\left(\int_{a}^{b}\left(\int_{t}^{b} h_{n-m-1}(t, \sigma(\tau)) \Delta \tau\right) \Delta t\right),\left(\int_{a}^{b}\left(\int_{a}^{t} h_{n-m-1}(t, \sigma(\tau)) \Delta \tau\right) \Delta t\right)\right\}$

and

3) assuming $f^{\Delta^{k+m}}(a)=f^{\Delta^{k+m}}(b)=0, k=1, \ldots, n-m-1$, from (3.34) we obtain

$$
\left|\int_{a}^{b} f^{\Delta^{m}}(t) \Delta t-\left[f^{\Delta^{m}}(a)(x-a)+f^{\Delta^{m}}(b)(b-x)\right]\right| \leq\left\|f^{\Delta^{n}}\right\|_{\infty,[a, b] \cap \mathbb{T}} .
$$




$$
\left[\left(\int_{a}^{x}\left(\int_{a}^{t} h_{n-m-1}(t, \sigma(\tau)) \Delta \tau\right) \Delta t\right)+\left(\int_{x}^{b}\left(\int_{t}^{b} h_{n-m-1}(t, \sigma(\tau)) \Delta \tau\right) \Delta t\right)\right]
$$

$\forall x \in[a, b] \cap \mathbb{T}$.

Proof. As in the proof of Theorem 3.1, now using Corollary 2.15 (4).

We give the following theorem.

Theorem 3.5. Let $f \in C_{r d}^{n}(\mathbb{T}), m, n \in \mathbb{N}, m<n, n-m$ is odd, $a, b \in \mathbb{T} ; a \leq b$. Then 1)

$$
\begin{aligned}
& \left|\int_{a}^{b} f^{\Delta^{m}}(t) \Delta t-\sum_{k=0}^{n-m-1}\left(f^{\Delta^{k+m}}(a) h_{k+1}(x, a)-f^{\Delta^{k+m}}(b) h_{k+1}(x, b)\right)\right| \leq \\
& \left\|f^{\Delta^{n}}\right\|_{L_{1}([a, b] \cap \mathbb{T})}\left\{\int_{a}^{x}(t-\sigma(a))^{n-m-1} \Delta t+\int_{x}^{b}(\sigma(b)-t)^{n-m-1} \Delta t\right\},
\end{aligned}
$$

$\forall x \in[a, b] \cap \mathbb{T}$,

2) assuming $f^{\Delta^{k+m}}(a)=f^{\Delta^{k+m}}(b)=0, k=0,1, \ldots, n-m-1$, we get from (3.40) that

$$
\begin{gathered}
\left|\int_{a}^{b} f^{\Delta^{m}}(t) \Delta t\right| \leq\left\|f^{\Delta^{n}}\right\|_{L_{1}([a, b] \cap \mathbb{T})} . \\
\left\{\int_{a}^{x}(t-\sigma(a))^{n-m-1} \Delta t+\int_{x}^{b}(\sigma(b)-t)^{n-m-1} \Delta t\right\},
\end{gathered}
$$

$\forall x \in[a, b] \cap \mathbb{T}$,

21) when $x=a$ by (3.41) we get

$$
\left|\int_{a}^{b} f^{\Delta^{m}}(t) \Delta t\right| \leq\left\|f^{\Delta^{n}}\right\|_{L_{1}([a, b] \cap \mathbb{T})}\left(\int_{a}^{b}(\sigma(b)-t)^{n-m-1} \Delta t\right),
$$

$2_{2}$ ) when $x=b$ by (3.41) we get

$$
\left|\int_{a}^{b} f^{\Delta^{m}}(t) \Delta t\right| \leq\left\|f^{\Delta^{n}}\right\|_{L_{1}([a, b] \cap \mathbb{T})}\left(\int_{a}^{x}(t-\sigma(a))^{n-m-1} \Delta t\right),
$$

$22_{3}$ ) by (3.42), (3.43) we have

$$
\begin{gathered}
\left|\int_{a}^{b} f^{\Delta^{m}}(t) \Delta t\right| \leq\left\|f^{\Delta^{n}}\right\|_{L_{1}([a, b] \cap \mathbb{T})} \cdot \\
\min \left\{\left(\int_{a}^{b}(\sigma(b)-t)^{n-m-1} \Delta t\right),\left(\int_{a}^{b}(t-\sigma(a))^{n-m-1} \Delta t\right)\right\},
\end{gathered}
$$

and 
obtain

3) assuming $f^{\Delta^{k+m}}(a)=f^{\Delta^{k+m}}(b)=0, k=1, \ldots, n-m-1$, from (3.40) we

$$
\begin{gathered}
\left|\int_{a}^{b} f^{\Delta^{m}}(t) \Delta t-\left[f^{\Delta^{m}}(a)(x-a)+f^{\Delta^{m}}(b)(b-x)\right]\right| \leq \\
\left\|f^{\Delta^{n}}\right\|_{L_{1}([a, b] \cap \mathbb{T})}\left\{\int_{a}^{x}(t-\sigma(a))^{n-m-1} \Delta t+\int_{x}^{b}(\sigma(b)-t)^{n-m-1} \Delta t\right\},
\end{gathered}
$$

$\forall x \in[a, b] \cap \mathbb{T}$.

Proof. As in Theorem 3.2, now using Corollary 2.15 (4).

We also give the next result.

Theorem 3.6. Let $f \in C_{r d}^{n}(\mathbb{T}), m, n \in \mathbb{N}, m<n, n-m$ is odd, $a, b \in \mathbb{T}$; $a \leq b$. Here $\sigma$ is continuous and $h_{n-m-1}(t, s)$ is jointly continuous. Let also $p, q>1: \frac{1}{p}+\frac{1}{q}=1$. Then

1)

$$
\begin{gathered}
\left|\int_{a}^{b} f^{\Delta^{m}}(t) \Delta t-\sum_{k=0}^{n-m-1}\left(f^{\Delta^{k+m}}(a) h_{k+1}(x, a)-f^{\Delta^{k+m}}(b) h_{k+1}(x, b)\right)\right| \leq \\
\left\|f^{\Delta^{n}}\right\|_{L_{q}([a, b] \cap \mathbb{T})}\left[\left(\int_{a}^{x}\left(\int_{a}^{t} h_{n-m-1}(t, \sigma(\tau))^{p} \Delta \tau\right)^{\frac{1}{p}} \Delta t\right)+\right. \\
\left.\left(\int_{x}^{b}\left(\int_{t}^{b} h_{n-m-1}(t, \sigma(\tau))^{p} \Delta \tau\right)^{\frac{1}{p}} \Delta t\right)\right]
\end{gathered}
$$

$\forall x \in[a, b] \cap \mathbb{T}$,

2) assuming $f^{\Delta^{k+m}}(a)=f^{\Delta^{k+m}}(b)=0, k=0,1, \ldots, n-m-1$, we get from (3.46) that

$$
\begin{aligned}
\left|\int_{a}^{b} f^{\Delta^{m}}(t) \Delta t\right| \leq & \left\|f^{\Delta^{n}}\right\|_{L_{q}([a, b] \cap \mathbb{T})}\left[\left(\int_{a}^{x}\left(\int_{a}^{t} h_{n-m-1}(t, \sigma(\tau))^{p} \Delta \tau\right)^{\frac{1}{p}} \Delta t\right)+\right. \\
& \left.\left(\int_{x}^{b}\left(\int_{t}^{b} h_{n-m-1}(t, \sigma(\tau))^{p} \Delta \tau\right)^{\frac{1}{p}} \Delta t\right)\right]
\end{aligned}
$$

$\forall x \in[a, b] \cap \mathbb{T}$

21) when $x=a$ we get from (3.47) that

$$
\left|\int_{a}^{b} f^{\Delta^{m}}(t) \Delta t\right| \leq\left\|f^{\Delta^{n}}\right\|_{L_{q}([a, b] \cap \mathbb{T})}\left(\int_{a}^{b}\left(\int_{t}^{b} h_{n-m-1}(t, \sigma(\tau))^{p} \Delta \tau\right)^{\frac{1}{p}} \Delta t\right),
$$


$2_{2}$ ) when $x=b$ we get from (3.47) that

$$
\left|\int_{a}^{b} f^{\Delta^{m}}(t) \Delta t\right| \leq\left\|f^{\Delta^{n}}\right\|_{L_{q}([a, b] \cap \mathbb{T})}\left(\int_{a}^{b}\left(\int_{a}^{t} h_{n-m-1}(t, \sigma(\tau))^{p} \Delta \tau\right)^{\frac{1}{p}} \Delta t\right),
$$

23) by (3.48), (3.49) we get

$$
\begin{gathered}
\left|\int_{a}^{b} f^{\Delta^{m}}(t) \Delta t\right| \leq\left\|f^{\Delta^{n}}\right\|_{L_{q}([a, b] \cap \mathbb{T})} \cdot \\
\min \left\{\left(\int_{a}^{b}\left(\int_{t}^{b} h_{n-m-1}(t, \sigma(\tau))^{p} \Delta \tau\right)^{\frac{1}{p}} \Delta t\right),\right. \\
\left.\left(\int_{a}^{b}\left(\int_{a}^{t} h_{n-m-1}(t, \sigma(\tau))^{p} \Delta \tau\right)^{\frac{1}{p}} \Delta t\right)\right\},
\end{gathered}
$$

and

3) assuming $f^{\Delta^{k+m}}(a)=f^{\Delta^{k+m}}(b)=0, k=1, \ldots, n-m-1$, we get from (3.46) that

$$
\begin{gathered}
\left|\int_{a}^{b} f^{\Delta^{m}}(t) \Delta t-\left[f^{\Delta^{m}}(a)(x-a)+f^{\Delta^{m}}(b)(b-x)\right]\right| \leq \\
\left\|f^{\Delta^{n}}\right\|_{L_{q}([a, b] \cap \mathbb{T})}\left[\left(\int_{a}^{x}\left(\int_{a}^{t} h_{n-m-1}(t, \sigma(\tau))^{p} \Delta \tau\right)^{\frac{1}{p}} \Delta t\right)+\right. \\
\left.\left(\int_{x}^{b}\left(\int_{t}^{b} h_{n-m-1}(t, \sigma(\tau))^{p} \Delta \tau\right)^{\frac{1}{p}} \Delta t\right)\right]
\end{gathered}
$$

$\forall x \in[a, b] \cap \mathbb{T}$.

Proof. As in Theorem 3.3, by using Corollary 2.15 (4).

\section{Applications}

We need the following.

Remark 4.1 (See [8]). i) When $\mathbb{T}=\mathbb{R}$, then $h_{k}(t, s)=\frac{(t-s)^{k}}{k !}, \forall k \in \mathbb{N}_{0}, \forall t, s \in \mathbb{R}$, $\sigma(t)=t, \int_{a}^{b} f(t) \Delta t=\int_{a}^{b} f(t) d t, f^{\Delta}(t)=f^{\prime}(t), f^{\Delta^{k}}=f^{(k)} ;$ rd-continuous corresponds to $f$ continuous. 
ii) When $\mathbb{T}=\mathbb{Z}, h_{k}(t, s)=\frac{(t-s)^{(k)}}{k !}, \forall k \in \mathbb{N}_{0}, \forall t, s \in \mathbb{Z}$, where $t^{(0)}=1$, $t^{(k)}=\prod_{i=0}^{k-1}(t-i)$ for $k \in \mathbb{N}, \sigma(t)=t+1$,

$$
\begin{gathered}
\int_{a}^{b} f(t) \Delta t=\sum_{t=a}^{b-1} f(t), \quad a<b, \\
f^{\Delta}(t)=f(t+1)-f(t)=\Delta f(t), \\
f^{\Delta^{k}}(t)=\Delta^{k} f(t)=\sum_{l=0}^{k}\left(\begin{array}{c}
k \\
l
\end{array}\right)(-1)^{k-l} f(t+l),
\end{gathered}
$$

rd-continuous $f$ corresponds to any $f$.

We also need the following.

Remark 4.2 (See $[1,8]$ ). Consider $q>1, q^{\mathbb{Z}}=\left\{q^{k}: k \in \mathbb{Z}\right\}$, and the time scale $\mathbb{T}=q^{\overline{\mathbb{Z}}}=q^{\mathbb{Z}} \cup\{0\}$, which is very important in $q$-difference equations.

It holds that

$$
\begin{gathered}
h_{k}(t, s)=\prod_{\nu=0}^{k-1} \frac{t-q^{\nu} s}{\sum_{\mu=0}^{\nu} q^{\mu}}, \quad \forall s, t \in \mathbb{T} ; \\
\sigma(t)=q t, \quad \rho(t)=\frac{t}{q}, \quad \forall t \in \mathbb{T}, \\
f^{\Delta}(t)=\frac{f(q t)-f(t)}{(q-1) t}, \forall t \in \mathbb{T}-\{0\}, \\
f^{\Delta}(0)=\lim _{s \rightarrow 0} \frac{f(s)-f(0)}{s} .
\end{gathered}
$$

Next we give applications of our initial main results.

Theorem 4.3. Let $f \in C^{n}([a, b]), n \in \mathbb{N}$ is odd and $[a, b] \subset \mathbb{R}$. Then

$$
\begin{gathered}
\left|\int_{a}^{b} f(t) d t-\sum_{k=0}^{n-1} \frac{1}{(k+1) !}\left(f^{(k)}(a)(x-a)^{k+1}+(-1)^{k} f^{(k)}(b)(b-x)^{k+1}\right)\right| \\
\leq \frac{\left\|f^{(n)}\right\|_{\infty,[a, b]}}{(n+1) !}\left[(x-a)^{n+1}+(b-x)^{n+1}\right],
\end{gathered}
$$

$\forall x \in[a, b]$.

Proof. By Theorem 3.1, (3.1).

We continue with the following. 
Theorem 4.4. Let $f \in C^{n}([a, b]), n \in \mathbb{N}$ is odd, $[a, b] \subset \mathbb{R}$. Then

$$
\begin{gathered}
\left|\int_{a}^{b} f(t) d t-\sum_{k=0}^{n-1} \frac{1}{(k+1) !}\left(f^{(k)}(a)(x-a)^{k+1}+(-1)^{k} f^{(k)}(b)(b-x)^{k+1}\right)\right| \\
\leq \frac{\left\|f^{(n)}\right\|_{L_{1}([a, b])}}{n}\left[(x-a)^{n}+(b-x)^{n}\right]
\end{gathered}
$$

$\forall x \in[a, b]$.

Proof. By Theorem 3.2, (3.16).

We also give the following.

Theorem 4.5. Let $f \in C^{n}([a, b]), n \in \mathbb{N}$ is odd and $[a, b] \subset \mathbb{R}$. Let also $p, q>1$ : $\frac{1}{p}+\frac{1}{q}=1$. Then

$$
\begin{gathered}
\left|\int_{a}^{b} f(t) d t-\sum_{k=0}^{n-1} \frac{1}{(k+1) !}\left(f^{(k)}(a)(x-a)^{k+1}+(-1)^{k} f^{(k)}(b)(b-x)^{k+1}\right)\right| \\
\leq \frac{\left\|f^{(n)}\right\|_{L_{q}([a, b])}}{(n-1) !(p(n-1)+1)^{\frac{1}{p}}\left(n+\frac{1}{p}\right)}\left[(x-a)^{n+\frac{1}{p}}+(b-x)^{n+\frac{1}{p}}\right],
\end{gathered}
$$

$\forall x \in[a, b]$.

Proof. By Theorem 3.3, (3.25).

We continue with the following.

Theorem 4.6. Let $f: \mathbb{Z} \rightarrow \mathbb{R}$, $n$ is an odd number, $a, b \in \mathbb{Z}$; $a \leq b$. Then

$$
\begin{gathered}
\left|\sum_{t=a}^{b-1} f(t)-\sum_{k=0}^{n-1} \frac{1}{(k+1) !}\left(\Delta^{k} f(a)(x-a)^{(k+1)}-\Delta^{k} f(b)(x-b)^{(k+1)}\right)\right| \leq \\
\frac{\left\|\Delta^{n} f\right\|_{\infty,[a, b] \cap \mathbb{Z}}}{(n-1) !}\left[\left(\sum_{t=a}^{x-1}\left(\sum_{\tau=a}^{t-1}(t-\tau-1)^{(n-1)}\right)\right)+\left(\sum_{t=x}^{b-1}\left(\sum_{\tau=t}^{b-1}(t-\tau-1)^{(n-1)}\right)\right)\right],
\end{gathered}
$$

$\forall x \in[a, b] \cap \mathbb{Z}$.

Proof. By Theorem 3.1, (3.1), see also Remark 4.1 (ii).

We give the next result. 
Theorem 4.7. Let $f: \mathbb{Z} \rightarrow \mathbb{R}, n \in \mathbb{N}$ is odd, $a, b \in \mathbb{Z} ; a \leq b$. Then

$$
\begin{gathered}
\left|\sum_{t=a}^{b-1} f(t)-\sum_{k=0}^{n-1} \frac{1}{(k+1) !}\left(\Delta^{k} f(a)(x-a)^{(k+1)}-\Delta^{k} f(b)(x-b)^{(k+1)}\right)\right| \leq \\
\left(\sum_{t=a}^{b-1}\left|\Delta^{n} f(t)\right|\right)\left\{\sum_{t=a}^{x-1}(t-a-1)^{n-1}+\sum_{t=x}^{b-1}(b+1-t)^{n-1}\right\},
\end{gathered}
$$

$\forall x \in[a, b] \cap \mathbb{Z}$.

Proof. By Theorem 3.2, (3.16) and Remark 4.1 (ii).

We give the following result.

Theorem 4.8. Let $f: \mathbb{Z} \rightarrow \mathbb{R}$, $n$ is an odd number, $a, b \in \mathbb{Z} ; a \leq b$, let also $p, q>1$ : $\frac{1}{p}+\frac{1}{q}=1$. Then

$$
\begin{gathered}
\left|\sum_{t=a}^{b-1} f(t)-\sum_{k=0}^{n-1} \frac{1}{(k+1) !}\left(\Delta^{k} f(a)(x-a)^{(k+1)}-\Delta^{k} f(b)(x-b)^{(k+1)}\right)\right| \leq \\
\frac{\left(\sum_{t=a}^{b-1}\left|\Delta^{n} f(t)\right|^{q}\right)^{\frac{1}{q}}}{(n-1) !}\left[\left(\sum_{t=a}^{x-1}\left(\sum_{\tau=a}^{t-1}\left((t-\tau-1)^{(n-1)}\right)^{p}\right)^{\frac{1}{p}}\right)+\right. \\
\left.\left(\sum_{t=x}^{b-1}\left(\sum_{\tau=t}^{b-1}\left((t-\tau-1)^{(n-1)}\right)^{p}\right)^{\frac{1}{p}}\right)\right]
\end{gathered}
$$

$\forall x \in[a, b] \cap \mathbb{Z}$.

Proof. By Theorem 3.3, (3.25) and Remark 4.1 (ii).

We continue with the following theorem.

Theorem 4.9. Let $f \in C_{r d}^{n}\left(q^{\overline{\mathbb{Z}}}\right), n \in \mathbb{N}$ is odd, $a, b \in q^{\overline{\mathbb{Z}}} ; a \leq b$. Then

$$
\begin{gathered}
\left|\int_{a}^{b} f(t) \Delta t-\sum_{k=0}^{n-1}\left(f^{\Delta^{k}}(a) \prod_{\nu=0}^{k} \frac{x-q^{\nu} a}{\sum_{\mu=0}^{\nu} q^{\mu}}-f^{\Delta^{k}}(b) \prod_{\nu=0}^{k} \frac{x-q^{\nu} b}{\sum_{\mu=0}^{\nu} q^{\mu}}\right)\right| \leq \\
\left\|f^{\Delta^{n}}\right\|_{L_{1}\left([a, b] \cap q^{\mathbb{Z}}\right)}\left\{\int_{a}^{x}(t-q a)^{n-1} \Delta t+\int_{x}^{b}(q b-t)^{n-1} \Delta t\right\},
\end{gathered}
$$

$\forall x \in[a, b] \cap q^{\overline{\mathbb{Z}}}$. 
Proof. By Theorem 3.2, (3.16), and Remark 4.2.

We finish with the next result.

Theorem 4.10. Let $f \in C_{r d}^{n}\left(q^{\overline{\mathbb{Z}}}\right), m, n \in \mathbb{N} ; m<n, n-m$ is odd, $a, b \in q^{\overline{\mathbb{Z}}} ; a \leq b$. Then

$$
\begin{aligned}
& \left|\int_{a}^{b} f^{\Delta^{m}}(t) \Delta t-\sum_{k=0}^{n-m-1}\left(f^{\Delta^{k+m}}(a) \prod_{\nu=0}^{k} \frac{x-q^{\nu} a}{\sum_{\mu=0}^{\nu} q^{\mu}}-f^{\Delta^{k+m}}(b) \prod_{\nu=0}^{k} \frac{x-q^{\nu} b}{\sum_{\mu=0}^{\nu} q^{\mu}}\right)\right| \leq \\
& \left\|f^{\Delta^{n}}\right\|_{L_{1}\left([a, b] \cap q^{\bar{Z}}\right)}\left\{\int_{a}^{x}(t-q a)^{n-m-1} \Delta t+\int_{x}^{b}(q b-t)^{n-m-1} \Delta t\right\},
\end{aligned}
$$

$\forall x \in[a, b] \cap q^{\overline{\mathbb{Z}}}$.

Proof. By Theorem 3.5, (3.40), and Remark 4.2.

One can give many similar applications for other time scales.

\section{References}

[1] R. Agarwal, M. Bohner, Basic Calculus on time scales and some of its applications, Results Math. 35(1999), no. 1-2, 3-22.

[2] R. Agarwal, M. Bohner, A. Peterson, Inequalities on time scales: a survey, Math. Inequalities \& Applications, Vol. 4, no. 4, (2001), 535-557.

[3] G.A. Anastassiou, Time Scales inequalities, Intern. J. of difference equations, 5 (1), 1-23 (2010).

[4] G. Anastassiou, Intelligent Mathematics: Computational Analysis, Springer, Heidelberg, New York, 2011.

[5] M. Bohner and G. Guisenov, The Convolution on time scales, Abstract and Applied Analysis, Vol. 2007, Article ID 58373, 24 pages.

[6] M. Bohner and B. Kaymakcalan, Opial inequalities on time scales, Ann. Polon. Math. 77 (2001), no. 1, 11-20.

[7] M. Bohner and T. Matthews, Ostrowski inequalities on time scales, JIPAM, J. Inequal. Pure Appl. Math. 9 (2008), no. 1, Article 6, 8 pp.

[8] M. Bohner and A. Peterson, Dynamic equations on time scales: An Introduction with Applications, Birkhäuser, Boston (2001). 
[9] R. Higgins and A. Peterson, Cauchy functions and Taylor's formula for Time scales $\mathbb{T}$, (2004), in Proc. Sixth. Internat. Conf. on Difference equations, edited by B. Aulbach, S. Elaydi, G. Ladas, pp. 299-308, New Progress in Difference Equations, Augsburg, Germany, 2001, publisher: Chapman \& Hall / CRC.

[10] S. Hilger, Ein Maßketten-Kalkül mit Anwendung auf Zentrumsmannigfaltigkeiten, PhD. thesis, Universität Würzburg, Germany (1988).

[11] K.S.K. Iyengar, Note on an inequality, Math. Student, 6 (1938), 75-76. 